\title{
Nanofluids, Synthesis and Stability - Brief Review
}

\section{Nanofluidos, Sintesis y Estabilidad - Breve Revision}

\author{
Jorge Silva-Yumi ${ }^{1,2}$, Telmo Moreno Romero ${ }^{2,3}$, and Gabriela Chango Lescano ${ }^{2,3}$ \\ ${ }^{1}$ Escuela Superior Politécnica de Chimborazo, Facultad de Ciencias, Química, Riobamba, \\ Ecuador \\ ${ }^{2}$ Grupo de Investigacion y Desarrollo Tecnologico de Energias Renovables.(GIDETER), \\ Riobamba, Ecuador \\ ${ }^{3}$ Escuela Superior Politécnica de Chimborazo, Facultad de Mecánica, Mecánica, Riobamba, \\ Ecuador
}

I International Congress of

Morona Santiago-CICTMS 2020

Corresponding Author:

Jorge Silva-Yumi

jorge.silvay@espoch.edu.ec

Published: 29 August 2021

Production and Hosting by

Knowledge $E$

(c) Jorge Silva-Yumi et al. This article is distributed under the terms of the Creative Commons Attribution License, which permits unrestricted use and redistribution provided that the original author and source are credited.

\section{Abstract}

Nanofluids constitute an alternative for the most efficient use of energy as they allow generating or improving thermal properties among others of traditional fluids, they are defined as so-called base fluids, such as: water, ethylene glycol, oils, etc., which contain nanoparticles in suspension , such as: aluminum oxide, silicon oxide, titanium oxide, metal nanoparticles, carbon nanotubes, graphene, carbides, etc. Nanofluids can be synthesized by two methods, the nanoparticles can be obtained separately and then the nanofluid is prepared or both nanoparticles and the nanofluid can be prepared simultaneously, an important factor to consider in obtaining nanofluids is their stability. Stability can be achieved by physical treatment or chemical treatment using surfactants. There are many studies about nanofluids, however, most are obtained with synthetic nanoparticles, leaving the use of natural nanoparticles as a field to be explored, as well as other surfactants to improve their stability.

Keywords: nanofluids, hybrid nanofluids, nanoparticles, nano refrigerant

\section{Resumen}

Los nanofluidos constituyen una alternativa para el uso más eficiente de energía pues permiten generar o mejorar las propiedades térmicas entre otras de los fluidos tradicionales, son definidos como fluidos denominados base, como: agua, etilenglicol, aceites, etc., que contienen nanopartículas en suspensión, como: óxido de aluminio, óxido de silicio, óxido de titanio, nanopartículas metálicas, nanotubos de carbono, grafeno, carburos, etc. Los nanofluidos se pueden sintetizar por dos métodos, se pueden obtener las nanopartículas por separado y luego preparar el nanofluido o se puede preparar simultáneamente las nanopartículas y el nanofluido, un factor importante a considerar en la obtención de nanofluidos es su estabilidad. La estabilidad se puede lograr mediante tratamiento físico o tratamiento químico mediante la utilización de surfactantes. Existen muchos estudios acerca de nanofluidos sin embargo, la mayoría se obtienen con nanopartículas sintéticas, quedando el uso de nanopartículas naturales como un campo por explorar al igual que otros surfactantes para mejorar su estabilidad.

Palabras Clave: nanofluidos, nonofluidos híbridos, nanoparticulas, nanorefrigerantes. 


\section{Introducción}

La preocupación mundial por el uso de una energía sostenible, que sea amigable con el ambiente, así como, su uso eficiente que no implique solo el ahorro sino la optimización de equipos y sistemas ha generado el desarrollo de los nanofluidos [1], los que permiten mejorar de alguna manera la eficiencia energética por las características que presentan, como: mejor conductividad térmica y mejor transferencia de calor [2] respecto a los fluidos convencionales.

Los nanofluidos son definidos como fluidos denominados base que contienen dispersas nanopartículas [3] metálicas, óxidos, nanotubos de carbono, grafeno, óxido de grafeno, etc.

Dentro de los nanofluidos se incluyen: nanolubricantes que corresponden a lubricantes producidos por la dispersión de nanopartículas en aceites lubricantes convencionales [4], nanorefrigerantes, que son también mezclas estables de nanopartículas y refrigerantes enfocados en mejorar las características de transferencia de calor [5]

Los nanofluidos se usan por sus beneficios en la transferencia de calor en refrigeración de motores, colectores solares, sistemas térmicos fotovoltaicos, refrigeración de componentes electrónicos, en el área de alimentos en unidades de procesamiento térmico, etc.

El objetivo de esta investigación es realizar una revisión sintética de los nanofluidos, determinar el tipo de nanopartículas utilizadas en su síntesis, así como, los fluidos base y los surfactantes utilizados para lograr una mejor estabilidad de las nanopartículas dispersas con el fin de establecer nuevas áreas de investigación.

\subsection{Metodología}

La revisión se realizó durante el periodo 2019-2020, se utilizó la base de datos ScienceDirect para la búsqueda de los artículos de revisión y de investigación mediante la utilización de las palabras clave: nanofluids, hybrid nanofluids y nanorefrigerants, review nanofluids, review hybrid nanofluids y review nanorefrigerants.

Se seleccionaron 43 artículos a partir de los cuales se ha sintetizado y sistematizado la información que se presenta en la presente revisión. Se inicia con la definición de los nanofluidos y nanofluidos hibridos, posteriormente se presentan los métodos de síntesis, así como, las nanopartículas y fluidos base utilizados, finalmente se menciona los tensoactivos que permiten lograr una mejor estabilidad de los nanofluidos.

\subsection{Desarrollo y Discusión}

Los nanofluidos son suspensiones estables y uniformes constituidos por fluidos denominados base, como: agua, etilenglicol, aceites, etc., que contienen dispersas nanopartículas [3]. Las nanopartículas utilizadas son óxidos de: aluminio [4], cobre, titanio, zirconio, wolframio, zinc, silicio [6], nanopartículas metálicas de cobre, plata, oro, hierro [7], 
nanotubos de carbono [8], grafeno funcionalizado [9] óxido de grafeno reducido [10] óxido de galio [11], óxido de zinc [12], entre otras.

Los nanofluidos muestran propiedades térmicas significativamente mejores que las de los fluidos base. Las nanopartículas ayudan a mejorar la conductividad térmica, debido a que cambian notablemente las propiedades de transporte y las características de transferencia de calor de la suspensión [13], el coeficiente de transferencia de calor [10], así como, la eficiencia térmica [14] sin embargo, una de las desventajas de los nanofluidos es que exhiben altas viscosidades debido a la presencia de las nanopartículas, lo que requiere mayor potencia de bombeo en los equipos.

Los nanofluidos han encontrado aplicaciones relevantes en procesos de transferencia de calor de varios sistemas, han sido empleados en colectores solares [8, 10], intercambiadores de calor, sistemas fotovoltaicos térmicos $[15,16]$, sistemas de aire acondicionado [17], conductividad térmica [18], enfriamiento de chips electrónicos, motores de automóviles (como lubricantes y refrigerantes), refrigeradores y plantas de energía nuclear (como refrigerantes para barras de combustible nuclear) [19].

\subsection{Nanofluidos Híbridos}

Son una nueva clase de nanofluídos que contienen dos o incluso tres diferentes tipos de nanopartículas en un fluido base [6].

Al igual que en los nanofluidos simples se utilizan diferentes nanoparticulas para su elaboración [20], entre las combinaciones de nanopartículas utilizadas se pueden mencionar Ag-MWCNT (plata - nanotubos de pared múltiple) [21] SWCNT-MgO (nanotubos de pared simple - óxido de magnesio), MWCNT-MgO, MWCNT-Fe $\mathrm{O}_{4}, \mathrm{Fe}_{3} \mathrm{O}_{4}$-grafeno, $\mathrm{Al}_{2} \mathrm{O}_{3}-\mathrm{Cu}, \mathrm{Al}_{2} \mathrm{O}_{3}-\mathrm{Ag}$, [22], $\mathrm{Cu}-\mathrm{TiO}_{2}$, [23].

A diferencia de los fluidos convencionales como aceite, agua y glicoles, así como, de los nanofluidos basados en un solo tipo de nanopartículas, los nanofluidos híbridos presentan mejores propiedades termofísicas, así como, un mejor rendimiento de transferencia de calor, especialmente en las áreas de automoción, electromecánica, procesos de fabricación y energía solar [6, 23].

\subsection{Preparación de Nanofluídos}

Los nanofluídos se preparan por dos métodos: el método de un paso y el método de dos pasos [24, 25].

\subsubsection{Método de un paso}

Implica la síntesis y dispersión de las nanopartículas en el fluido base de manera simultánea, en una sola etapa [26]. 


\subsubsection{Método de dos pasos}

Las nanopartículas son sintetizadas por cualquiera de los enfoques: botton up (de abajo hacia arriba); basado en métodos químicos y biológicos, o top down (de arriba hacia abajo); el cual implica procesos de miniaturización o descomposición de estructuras macroscópicas, posteriormente estas nanopartículas pueden ser dispersadas en cualquier fluido base [26].

En los dos métodos de preparación de nanofluidos es necesario asegurarse que las nanopartículas se encuentren dispersas homogéneamente, y que el nanofluido muestre características de estabilidad y las propiedades deseadas, para esto se realizan las pruebas de caracterización correspondiente como: medición de velocidad de sedimentación, medición del potencial zeta, medición de absorbancia o transmitancia y/o análisis por dispersión dinámica de la luz.

\subsection{Nanopartículas utilizadas para nanofluídos}

\subsubsection{Oxidos}

Son resistentes a la oxidación, son químicamente estables, la densidad de algunos es baja lo que permite su fácil dispersión en los fluidos base, como: agua, etilenglicol, aceites, etc., sin embargo, tienen una baja conductividad térmica si se los compara con los metales [27]. Se han utilizado óxido de aluminio o alúmina [28, 29] debido su alta conductividad térmica y baja densidad, oxido de calcio [30], óxido de galio [11], óxido de titanio, óxido de hierro [31, 32] óxido de cobre [33], óxido de zirconio, wolframio, zinc, silicio [6].

El óxido de silicio por ejemplo mejora significativamente el coeficiente de transferencia de calor del refrigerante R134a hasta $163.2 \%$ con una concentración de partículas de $0.4 \%$ [34]. Con óxido de hierro se obtiene nanofluidos magnéticos ya que las nanopartículas que los constituyen corresponden a nanopartículas magnéticas y por tanto responden a un campo magnético.

\subsubsection{Nanopartículas metálicas}

Los metales generalmente tienen una conductividad térmica más alta que los fluidos. Por lo tanto el uso de nanopartículas metálicas en un fluido produce un buen nanofluido termoconductor [8]

Se han empleado nanopartículas metálicas de cobre [35], plata [6, 7, 36] y oro [7]. En el caso de las partículas metálicas de plata que tienen una alta conductividad térmica, su conductividad eléctrica puede hacerlas no tan útiles para aplicaciones de enfriamiento electrónico [37]. 


\subsubsection{Materiales de carbono}

Se ha evaluado la utilización de nanotubos de carbono [8, 35], grafeno [38], óxido de grafeno reducido [10] incluso nanopartículas de diamante debido a su alta conductividad térmica (la más alta de cualquier material) y su insignificante conductividad eléctrica (0.01 nS/m) [37].

Nanotubos de carbono de paredes múltiples dispersos en agua destilada utilizados en un termosifón y un colector solar de placa plana de circulación forzada muestran una mejora de la eficiencia exergética y la eficiencia energética del sistema [8].

Un nano-refrigerante preparado con nanopartículas de grafeno tiene un coeficiente de fricción más bajo y una conductividad térmica más alta. La adición de $30 \mathrm{mg} / \mathrm{L}$ de grafeno a un refrigerante de un refrigerador doméstico incrementa la velocidad de enfriamiento del compartimento de alimentos frescos en un $5.6 \%$ y la velocidad de enfriamiento del congelador en un $4.7 \%$ [39].

Nanofluidos híbridos a base de carbón activado-óxido de grafeno/etilenglicol mostraron un aumento de la conductividad térmica en comparación con el fluido base, además la conductividad eléctrica también muestra un aumento significativo [38].

\subsubsection{Otras nanopartículas}

Se ha evaluado la utilización de nanocarburo de silicio ( $\mathrm{SiC}$ ) como aditivo para mejorar la conductividad térmica del nanofluido resultante a base de agua $[15,16]$, que se caracterizan por su alta conductividad térmica.

Se han utilizado también puntos cuánticos 'quantum dots' de silicio para la obtención de nanofluidos orientados a la extracción de petróleo obteniéndose una mayor [40].

\subsection{Fluidos base}

Agua, aceite, alcohol, querosene son algunos de los líquidos base utilizados para la elaboración de nanofluidos.

El agua y los glicoles son los líquidos base más estudiados, los glicoles como el etilenglicol y el propilenglicol tienen puntos de congelación más bajos que el agua y se utilizan solos o en mezcla con el agua. Por lo general se han utilizado tanto etilenglicol como propilenglicol [16] debido a que están disponibles de manera muy accesible y tienen costos razonables. A más del agua y los glicoles se utilizan también aceite de motor, querosene, aceite vegetal, parafina, entre otros.

Los fluidos convencionales de transferencia de calor, como el agua y el aceite, tienen baja conductividad térmica, lo que es un impedimento para mejorar la eficiencia de todos los intercambiadores de calor [15].

Como una alternativa a los lubricantes convencionales han surgido los bio lubricantes, que son menos tóxicos que los aceites minerales, sin embargo, las propiedades lubricantes de los bio-lubricantes son ligeramente menores que las de los aceites 
minerales y tienen una pobre estabilidad oxidativa que restringe su uso a temperaturas elevadas, el aceite de salvado de arroz [30] es uno de los fluidos base usado para obtener lubricantes biodegradables.

\subsubsection{Estabilidad}

Si bien los nanomateriales son considerados materiales con dimensiones en la escala de 1 a $100 \mathrm{~nm}$, dentro de este rango por los diferentes procesos de síntesis se pueden obtener diferentes tamaños de nanopartículas. La forma, el tipo y el tamaño de las nanopartículas son factores clave en la estabilidad de los nanofluídos y tienen un impacto directo en el período de estabilidad, así como, en el tipo y la naturaleza del líquido base utilizado [15].

El desafío en la preparación de nanofluidos es obtener una suspensión estable evitando la aglomeración de las nanopartículas, ya que la conductividad térmica depende de la estabilidad de la dispersión las nanopartículas adicionadas, la deposición de las nanopartículas reduce la conductividad térmica de los nanofluidos y por tanto la transferencia de calor [15].

Es necesario que las nanopartículas permanezcan en suspensión por un considerable lapso de tiempo, sin embargo, debido a su gran área superficial e inestabilidad por efecto de la no saturación de los átomos más externos [41] estas tienen una alta tendencia a aglomerarse por acción de las fuerzas de Van der Waals [8], especialmente cuando estas dominan sobre las fuerzas de repulsión. La agregación de las nanopartículas puede provocar la posterior sedimentación y generar problemas durante la operación de los equipos en los cuales se utilicen estos nanofluidos.

La variable clave para lograr un nanofluido estable es evitar la aglomeración, la cual se puede lograr mediante tratamiento físico o químico. En el tratamiento físico la dispersión se puede lograr de manera mecánica a través del uso de ultrasonidos [37], sin embargo, se ha demostrado que el uso de vibración ultrasónica para formar una suspensión estable de nanopartículas puede producir una suspensión que no es completamente estable [15], otra manera de lograr la dispersión es variar o generar carga superficial en las nanoparticulas de tal manera que se produzca la repulsión por carga evitando la aglomeración.

La dispersión química se realiza mediante la adición de surfactantes o polímeros $[19,37]$ o mediante la variación del pH del medio. La adición de surfactantes (Tabla 1) permite mejorar la estabilidad de las nanopartículas y por tanto la conductividad térmica y la transferencia de calor [19].

Los tensioactivos son generalmente compuestos orgánicos que reducen la tensión superficial entre dos líquidos o un líquido y un sólido, la estabilidad del nanofluido depende del tensioactivo utilizado [15].

Si bien la tendencia es que la adición de surfactantes en los nanofluidos conduce a una mejora en su estabilidad, la presencia de estos puede también disminuir la conductividad térmica $[15,19]$, en especial cuando se aumenta su concentración debido a que se forma una capa delgada sobre las nanopartículas que dificulta el rendimiento de la transferencia de calor del nanofluido [19]. 


\section{Table 1}

Surfactantes utilizados para estabilizar nanopartículas en suspensión.

\begin{tabular}{lll}
\hline Nombre & Ref. \\
\hline Goma Arábica & {$[21]$} \\
\hline Oleato de potasio & {$[36]$} \\
\hline Ácido oleico & {$[32]$} \\
\hline Dodecil betaína & {$[42]$} \\
\hline Dodecil sulfato de sodio (SDS) & {$[15,19,42]$} \\
\hline Dodecilbenceno sulfonato de sodio & {$[15,42]$} \\
\hline Salicilato de sodio & {$[21]$} \\
\hline Desoxicolato de sodio & {$[15]$} \\
\hline Bromuro de cetil trimetil amonio & {$[15]$} \\
\hline Cloruro de cetil trimetil amonio & {$[21]$} \\
\hline Bromuro de dodecil trimetilamonio & {$[11]$} \\
\hline Polivinilpirrolidona & {$[11]$} \\
\hline Cetil cetil amonio & {$[15]$} \\
\hline Desoxicolato de sodio & {$[15]$} \\
\hline Triton X-100 & {$[43]$} \\
\hline Polisorbato 20 & {$[19,43]$} \\
Polisorbato 80 & {$[43]$} \\
\hline Dibromuro de 1,2-bis (cetiltrimetilamonio) etano Dibromuro de 1,3-bis (cetildimetilamonio) & {$[7]$} \\
propano Dibromuro de 1,4-bis (cetildimetilamonio) butano Dibromuro de 1,6-bis & \\
(cetildimetilamonio) hexano Dibromuro de 1,8-bis (cetildimetilamonio) octano & \\
\hline
\end{tabular}

Por otra parte el uso de tensoactivo está restringido a aplicaciones de baja temperatura porque a alta temperatura, se pueden formar burbujas que afectan significativamente la eficiencia de transferencia de calor [8].

\section{Conclusiones}

Efectivamente los nanofluidos simples o híbridos constituyen una alternativa para lograr una mayor eficiencia energética, es necesario su comprensión y la ampliación de experiencias, si bien existe un amplio espectro de nanomateriales utilizados en la obtención de nanofluidos, su naturaleza es principalmente sintética. No se encontró la utilización de nanopartículas naturales como alofán, imogolita, halloysita o arcillas, por lo que se puede establecer que existe un campo de estudio aún por explorar. De la misma manera en el caso de los surfactantes queda un amplio número de compuestos tanto sintéticos como naturales a evaluar.

\section{Agradecimiento}

Al Instituto de Investigaciones de la Escuela Superior Politécnica de Chimborazo. 


\section{Conflicto de Intereses}

No existen intereses particulares por parte de los autores.

\section{References}

[1] Azmi WH, Sharif MZ, Yusof TM, Mamat R, Redhwan AAM. Potential of nanorefrigerant and nanolubricant on energy saving in refrigeration system - A review. Renew Sustain Energy Rev [Internet]. 2017;69(October 2016):415-28. dx.doi.org/10.1016/j.rser.2016.11.207

[2] Chakraborty S, Panigrahi PK. Stability of nanofluid: A review. Appl Therm Eng. 2020;174(March).

[3] Abbas F, Raza T, Babar H, Mansoor M, Sajjad U, Amer M. Nano fluid: Potential evaluation in automotive radiator. J ofMolecular Liq. 2020;297.

[4] Sanukrishna SS, Jose PM. Thermal and rheological characteristics of refrigerant compressor oil with alumina nanoparticles-An experimental investigation. Powder Technol [Internet]. 2018;339:119-29. https://doi.org/10.1016/j.powtec.2018.08.003

[5] Sanukrishna S, Murukan M, Jose PM. An overview of experimental studies on nanorefrigerants: Recent research, development and applications. Int J Refrig. 2018;88:552-77.

[6] Che SNA, Mahmud JM, Aziz Japar WMA, Muhammad Al. A review on preparation methods, stability and applications of hybrid nanofluids. Renew Sustain Energy Rev [Internet]. 2017;80(August):1112-22. http://dx.doi.org/10.1016/j.rser.2017.05.221

[7] Li D, Fang W, Feng Y, Geng Q, Song M. Stability properties of water-based gold and silver nanofluids stabilized by cationic gemini surfactants. J Taiwan Inst Chem Eng. 2019;97:458-65.

[8] Eltaweel M, Abdel-Rehim AA. Energy and exergy analysis of a thermosiphon and forced- circulation flatplate solar collector using MWCNT/Water nano fluid. Case Stud Therm Eng. 2019;14(February):100416.

[9] Paul G, Shit S, Hirani H, Kuila T, Murmu NC. Tribological behavior of dodecylamine functionalized graphene nanosheets dispersed engine oil nanolubricants. Tribol Int [Internet]. 2019;131:605-19. https: //doi.org/10.1016/j.triboint.2018.11.012

[10] Xu X, Xu C, Liu J, Fang X, Zhang Z. A direct absorption solar collector based on a water-ethylene glycol based nanofluid with anti-freeze property and excellent dispersion stability. Renew Energy. 2019;133:760-9.

[11] El-salamony RA, Morsi RE. Stable gallium oxide@ silica/polyvinyl pyrrolidone hybrid nanofluids: Preparation, characterization, and photo-activity toward removal of malachite green dye. J Mol Liq. 2018;271(1):589-98.

[12] Kumar M, Sawhney N, Sharma AK, Sharma M. Thermo-physical profile of zinc oxide nanoparticles dispersed in aqueous solution of propylene glycol. J Mol Liq [Internet]. 2018;249:650-8. http://dx.doi. org/10.1016/j.molliq.2017.11.095

[13] Said Z, Saidur R. Thermophysical properties of metal oxides nanofluids. Nanofluids Thermophys Prop Met Oxides Nanofluids. 2017;

[14] Liu Z, Yan Y, Fu R, Alsaady M. Enhancement of solar energy collection with magnetic nano fluids. Therm Sci Eng Prog. 2018;8(March):130-5.

[15] AI-Waeli AHA, Chaichan MT, Kazem HA, Sopian K. Evaluation and analysis of nanofluid and surfactant impact on photovoltaic-thermal systems. Case Stud Therm Eng. 2019;13(November 2018):100392.

[16] AI-Waeli AHA, Chaichan MT, Sopian K, Kazem HA. Influence of the base fluid on the thermo-physical properties of PV/T nanofluids with surfactant. Case Stud Therm Eng [Internet]. 2019;13. https://doi.org/ 10.1016/j.csite.2018.10.001

[17] Ganvir RB, Walke P V, Kriplani VM. Heat transfer characteristics in nanofluid - A review. Renew Sustain Energy Rev. 2017;75(November 2016):451-60.

[18] Taw MM. Experimental studies of nanofluid thermal conductivity enhancement and applications: A review. Renew Sustain Energy Rev. 2017;75(November 2016):1239-53.

[19] Chakraborty S, Sengupta I, Sarkar I, Pal SK, Chakraborty S. Effect of surfactant on thermo-physical properties and spray cooling heat transfer performance of Cu-Zn-Al LDH nanofluid. Appl Clay Sci. 2019;168(June 2018):43-55.

[20] Ranga BJA, Kumar KK, Srinivasa RS. State-of-art review on hybrid nanofluids. Renew Sustain Energy Rev. 2017;77(April):551-65.

[21] Sun B, Zhang Y, Yang D, Li H. Experimental study on heat transfer characteristics of hybrid nano fluid impinging jets. Appl Therm Eng. 2019;151(July 2018):556-66.

[22] Huminic G, Huminic A. Hybrid nanofluids for heat transfer applications - A state-of-the-art review. Int J Heat Mass Transf [Internet]. 2018;125:82-103. https://doi.org/10.1016/j.ijheatmasstransfer.2018.04.059 
[23] Sundar LS, Sharma K V., Singh MK, Sousa ACM. Hybrid nanofluids preparation, thermal properties, heat transfer and friction factor - A review. Renew Sustain Energy Rev [Internet]. 2017;68(August 2016):18598. http://dx.doi.org/10.1016/j.rser.2016.09.108

[24] Leong KY, Ahmad KZK, Chyuan H, Ghazali MJ, Baharum A. Synthesis and thermal conductivity characteristic of hybrid nano fluids - A review. Renew Sustain Energy Rev. 2017;75(May 2015):868-78.

[25] Dhinesh KD, Valan AA. A comprehensive review of preparation, characterization, properties and stability of hybrid nanofluids. Renew Sustain Energy Rev [Internet]. 2018;81(August 2016):1669-89. https://doi. org/10.1016/j.rser.2017.05.257

[26] Babar H. Towards hybrid nano fluids: Preparation, thermophysical properties, applications, and challenges. J ofMolecular Liq. 2019;281:598-633.

[27] Suganthi K, Rajan K. Metal oxide nano fluids: Review of formulation, thermo-physical properties, mechanisms, and heat transfer performance. Renew Sustain Energy Rev. 2017;76(February):226-55.

[28] Saghir MZ, Ahadi A, Mohamad A, Srinivasan S. Water aluminum oxide nanofluid benchmark model. Int J Therm Sci. 2016;109:148-58.

[29] Ho CJ, Liao J, Li C, Yan W, Amani M. Experimental study of cooling characteristics of water-based alumina nano fluid in a minichannel heat sink. Case Stud Therm Eng. 2019;14(February):1-9.

[30] Sunil J, Vignesh J, Vettumperumal R, Maheswaran R, Raja RAA. The thermal properties of CaONanofluids. Vacuum. 2019;161(December 2018):383-8.

[31] Goharkhah M, Ashjaee M, Shahabadi M. Experimental investigation on convective heat transfer and hydrodynamic characteristics of magnetite nano fluid under the influence of an alternating magnetic field. 2016;99:113-24.

[32] Agnihotri P, Lad VN. Magnetic nanofluid: synthesis and characterization. Chem Pap [Internet]. 2020;74(9):3089-100. https://doi.org/10.1007/s11696-020-01138-w

[33] Khan MS, Abid M, Ali HM, Amber KP, Bashir MA, Javed S. Comparative performance assessment of solar dish assisted s-CO2 Brayton cycle using nanofluids. Appl Therm Eng [Internet]. 2019;148:295-306. https://doi.org/10.1016/j.applthermaleng.2018.11.021

[34] Sanukrishna SS, Shafi M, Murukan M, Jose PM. Effect of SiO2 nanoparticles on the heat transfer characteristics of refrigerant and tribological behaviour of lubricant. Powder Technol. 2019;356:39-49.

[35] Che SNA, Witri MNA, Mamat R. Recent advancement of nanofluids in engine cooling system. Renew Sustain Energy Rev. 2017;75(April 2015):137-44.

[36] Parametthanuwat T, Bhuwakietkumjohn N, Rittidech S, Ding Y. Experimental investigation on thermal properties of silver nanofluids. 2015;56:80-90.

[37] Mashali F, Languri EM, Davidson J et al. Thermo-physical properties of diamond nanofluids: A review. Int J Heat Mass Transf [Internet]. 2019;129:1123-35. https://doi.org/10.1016/j.ijheatmasstransfer.

[38] Yarmand H, Gharehkhani S, Shirazi SFS et al. Nanofluid based on activated hybrid of biomass carbon/graphene oxide: Synthesis, thermo-physical and electrical properties. Int Commun Heat Mass Transf [Internet]. 2016;72:10-5. http://dx.doi.org/10.1016/j.icheatmasstransfer.2016.01.004

[39] Yang S, Cui X, Zhou Y, Chen C. Study on the effect of graphene nanosheets refrigerant oil on domestic refrigerator performance. Int J Refrig [Internet]. 2020;110:187-95. https://doi.org/10.1016/j.ijrefrig.2019. 11.008

[40] Zhou Y, Wu X, Zhong X, Zhang S, Pu H, Zhao JX. Development of silicon quantum dots based nano-fluid for enhanced oil recovery in tight Bakken cores. Fuel. 2020;277(April).

[41] Sezer N, Atieh MA, Koç M. A comprehensive review on synthesis, stability, thermophysical properties, and characterization of nanofluids. Powder Technol [Internet]. 2019;344:404-31. https://doi.org/10.1016/ j.powtec.2018.12.016

[42] Kim S, Tserengombo B, Choi S, Noh J, Huh S. Experimental investigation of dispersion characteristics and thermal conductivity of various surfactants on carbon based nanomaterial. Int Commun Heat Mass Transf. 2018;91(December 2017):95-102.

[43] Gao T, Li C, Zhang Y et al. Dispersing mechanism and tribological performance of vegetable oil-based CNT nanofluids with different surfactants. Tribiology Int. 2019;131(August 2018):51-63. 